PARTIE I

Accountability,

gouvernance et efficacité 
INSEEC Business School

\section{Le comply or explain, un avatar de l'accountability}

En quoi la transposition du concept de comply or explain, dans de nombreux pays de I'Union européenne, et, en particulier en France, s'avère une forme dévoyée d'accountability ? Tel est l'objet de cet article transdisciplinaire, droit et gestion, relatif aux codes de gouvernance d'entreprise. La flexibilité dans la mise en œuvre de ce mécanisme apparaît inversement proportionnelle à la rigueur qu'exigerait un très haut niveau de transparence. L'évolution des pratiques démontre un certain conformisme qui fait courir le risque d'une perversion des principes : la logique du comply or explain tend, en effet, à substituer à l'obligation de rendre des comptes une option quant à l'application des normes privées de gouvernement des entreprises. 
$\mathrm{L}$ 'apparition en France, depuis une vingtaine d'années, des codes privés de gouvernement d'entreprise, venus des États-Unis, pour diffuser le modèle de la gouvernance actionnariale dans le droit des sociétés, interroge à nouveau sur les mutations de l'État, le rôle des nouvelles instances de régulation comme l'AMF (Autorité des marchés financiers) et le poids des acteurs économiques, tels les investisseurs institutionnels. Ainsi, une nouvelle catégorie de norme privée est née, très souple, qui s'élabore en dehors du processus législatif habituel, quoique reconnue par les pouvoirs publics, et ce, dans toute l'Union européenne. Le cas français retiendra plus particulièrement notre attention comme étant révélateur des tensions entre deux modèles historiques de gouvernance, actionnariale et partenariale, auxquels se superposent deux instruments juridiques très différents, la soft law et la hard law, qui, par la figure imposée du comply or explain (se conformer ou s'expliquer), s'entrechoquent dans le but de favoriser le modèle de gouvernance actionnariale. Imposée par la directive européenne 2006/46 du Parlement européen et du Conseil du 14 juin 2006, transposée par la loi no 2008-649 du 3 juillet 2008, la règle d'origine britannique du comply or explain destinée à permettre l'application de ces codes élaborés par des instances représentatives des entreprises, a été transposée en France de façon très alambiquée et atténuée par rapport au Royaume-Uni, puisque les sociétés françaises ont la possibilité, non seulement de déroger au code de gouvernance, mais aussi de ne pas l'appliquer $\mathrm{du}$ tout. Cette réplique dans notre droit est paradoxale puisque le législateur introduit d'une part, l'obligation d'appliquer un code de référence et d'autre part, la faculté de ne pas se référer à un code. De manière ubuesque, l'articulation entre ces nouveaux instruments du droit souple et la loi française - plus favorable à des dispositions de droit dur - a été traduite par une « obligation facultative $»$.

Le mécanisme du comply or explain n'est en fait qu'un avatar ${ }^{1}$ du principe d'accountability, dont il n'est au mieux qu'une traduction. En effet, idéalement ce mécanisme aurait pu être l'incarnation de l'accountability; dans les faits, l'avatar conduit à une transmutation du système normatif par inversion des valeurs, des principes et des normes.

Or, au-delà des codes de gouvernance pour lesquels son application était initialement requise, la méthode dite comply or explain s'étend progressivement à toutes les déclarations portant sur des données ESG (environnement, social, gouvernance) et devient l'outil de prédilection de l'accountability (ou responsabilité liée à l'obligation de rendre des comptes). Dans la nouvelle stratégie Europe 2020, 1'Union européenne " affirme l'existence d'un lien inextricable entre la responsabilité des entreprises et la gouvernance d'entreprise $»^{2}$.

1. Selon le Trésor de la Langue française Informatisé (CNRS éditions), le terme avatar apparaît pour la première fois en langue française au début du $\mathrm{XIX}^{\mathrm{e}}$ siècle, pour désigner l'incarnation d'une divinité hindoue, par extension le double d'un sujet ou d'un objet. Par analogie, une nouvelle acception du mot avatar est introduite en langue française (1822) afin de désigner les notions de changement, transformation ou métamorphose d'une personne ou d'une chose jusqu'à signifier à partir du $\mathrm{xx}^{\mathrm{e}}$ siècle les incidents, péripéties, incidents ou aléas d'un phénomène. 2. Résolution au Parlement européen du 6 février 2013 sur la responsabilité sociale des entreprises : promouvoir les intérêts de la société et ouvrir la voie à une reprise durable et inclusive (2012/2097) INI. 
Cette forme d'accountability, devenue la " pierre angulaire de la gouvernance " (IFA, 2013, p. 3 ; Poulle, 2011, p. 15) et, par extension, de la responsabilité sociale des entreprises (RSE), sera l'objet de notre étude. Nous démontrons ainsi que l'écueil majeur d'une transposition d'un outil décontextualisé, avec un choc des cultures juridiques, notamment en France, conduit à des transgressions (Babeau et Chanlat, 2008 ; Bréchet et al., 2008) : loin de la conformité, le conformisme tend à s'installer dans une spirale d'impunité, renforcée par le rôle subsidiaire désormais joué par l'État. Tout récemment, l'État français a ainsi renoncé à exercer un contrôle sur la gouvernance des entreprises privées. En contrepartie, l'Afep et le Medef viennent de réviser leur code de gouvernement d'entreprise des sociétés cotées, en particulier sur la question sensible des rémunérations des dirigeants (14 juin 2013) et de créer un haut comité de suivi de l'application de leur code. Or, si l'AMF, autorité publique, n'avait déjà que peu de pouvoir, ce haut comité quant à lui privé ne pourra en disposer davantage : émanation des organisations professionnelles privées (article 25-2 du Code Afep-Medef), son autorité est simplement morale. Non seulement l'État n'adoube plus la norme, mais il n'en est plus le garant.

La force normative dont se pare le concept de « comply or explain », notamment par le truchement du droit privé européen, peut conduire la gouvernance des entreprises, qu'il s'agisse du modèle actionnarial ou partenarial (Charreaux et Desbrières, 1998), dans une ornière, faute d'obligations contraignantes uniformisées, d'une part, et de réelles sanctions applicables, d'autre part.
L'analyse transdisciplinaire retenue en raison de l'objet même de cet article, entre sciences de gestion et sciences juridiques, nous invite, par son résultat, à réfléchir sur une question avant tout éthique, de responsabilité des organisations. Après avoir rappelé en quoi la logique de comply or explain est une facette du concept d'accountability et se rapporte historiquement et de façon intrinsèque aux codes de gouvernance, nous montrons que l'essaimage du concept comply or explain en Europe a accompagné la diffusion d'un modèle de gouvernance dominant à orientation actionnariale et ce, jusqu'en France, avant de soulever les principales pierres d'achoppement contre lesquelles risque de buter la gouvernance des entreprises par la mise en œuvre du comply or explain, qui de simple mécanisme a été érigé en véritable principe, en lieu et place de l'accountability.

\section{I - SE CONFORMER OU S'EXPLIQUER COMME MISE EN OEUVRE DE LACCOUNTABILITY}

\section{Comply or explain : une dimension de l'accountability}

Bien que la notion d'accountability revête plusieurs dimensions allant de la mesure de ce qui compte à la responsabilisation (Pesqueux, 2012), elle signifie, de façon principale, dans les textes en langue anglaise comme dans sa traduction en langue française, la responsabilité liée à l'obligation de rendre des comptes (Dumez, 2008). To account for, ou « rendre des comptes », rejoint l'accountability to, « être responsable de », selon les déclinaisons du Harrap's Shorter. Face à un concept en véritable extension (Mulgan, 2000), l'objet de cet article est d'explorer une des modali- 
tés de la mise en œuvre de l'accountability, le mécanisme du comply or explain (se conformer/appliquer ou expliquer), qui, à l'origine, a pour cadre de référence les codes de gouvernance (MacNeil et Li, 2006).

Le «mécanisme», issu du mot «mécanique » signifie «agencement de pièces diverses disposées pour produire un mouvement, un effet donné, tel le mécanisme d'une horloge » et, par association d'idées, en philosophie, «système d'explication de l'ensemble des phénomènes par le mouvement » (définition du Dictionnaire Quillet de la langue française, vol. 3, 1975). Cette définition du mécanisme correspond bien à l'image de la dynamique créée par le « comply or explain ». Sa structure interne, très originale, du fait de l'impression donnée à la fois d'une double contrainte ou d'une double faculté, permet d'expliquer les causes de son déploiement et de son évolution, comme fait social (Dumez, 2013). Puisque les entreprises ne sont pas tenues de suivre un code, elles ne peuvent qu'être incitées à se conformer à un code. Par un juste parallélisme des formes, la contrainte du « comply » est donc tempérée, également, par la possibilité de s'exonérer. Le succès du système repose sur l' " explain » perçu avant tout comme une autorisation préalable à l'autolimitation du principe d'accountability. C'est cette contrepartie de l'engagement de rendre des comptes qui forme un ensemble cohérent, un moyen de faire adhérer les entreprises à des règles de soft law. La finalité du mécanisme est d'obtenir que les entreprises entre dans une démarche vertueuse d'amélioration continue par une application plus rigoureuse de codes de gouvernance, qui comporteraient à l'avenir des exigences plus fortes et plus nombreuses.
Cette définition technique du mécanisme rejoint celle utilisée en sciences sociales pour rendre compte des phénomènes organisationnels et des comportements individuels (Hedström et Bearman, 2009). Elle permet surtout de comprendre, aux termes de notre recherche pourquoi la qualification de " principe d'action » retenue nous paraît plus juste que celle de «principe » relativement au «comply or explain », qui doit demeurer un moyen technique pour rendre des comptes, et non un principe, en lui-même, déconnecté de sa finalité. Pour la suite de nos développements, nous conservons, donc, à dessein, le mot « mécanisme » ainsi que les termes voisins (méthode, logique, outil, procédure, etc.), pour mieux approfondir ses effets positifs et négatifs. Le mécanisme de comply or explain repose sur une obligation de déclaration qui peut être doublée d'une obligation de rendre des comptes ou de s'expliquer en cas de non-conformité aux codes. Nous sommes donc en présence d'une forme de « matérialisation » du principe de transparence qui va au-delà du fait de se «mettre sous le regard des autres » (Pesqueux, 2012, p. 103) puisqu'elle inclut celui de répondre de ses décisions et actes devant autrui, premier pas vers la responsabilité. Qu'il s'agisse d'une capacité ou d'une obligation à rendre des comptes, la notion d'accountability est au cœur des codes de gouvernance (Weil, Gotshal \& Manges LLP, 2002 ; Wirtz, 2006). Si le verbe «comply» en anglais dénote à la fois le fait de se soumettre à des exigences particulières, et celui de satisfaire celles-ci, le verbe « se conformer »-retenu pour la traduction en langue française vise d'un point de vue juridique « l'action de se rendre semblable à la reproduction exacte d'un acte de référence »; quant au 
verbe « expliquer », il porte une signification similaire à celle du verbe « explain », celle de dérouler, mettre à plat et éclaircir (Poulle, 2011, p. 17).

Remarquons néanmoins que l'acte de conformité volontaire (compliance) aux recommandations présentes dans les codes relève plus de la contrainte que d'une pure et libre volonté. Le glissement qui s'est opéré, en une décennie, du vocable de « comply or explain » vers celui de « apply or explain » témoigne d'une extension ou d'un durcissement de la contrainte. C'est la résultante du couplage des obligations. Il s'agit tout aussi bien de fournir des informations, d'en apporter l'exactitude, que de se conformer aux recommandations ou de rendre des comptes d'un non-respect de ces dernières. Telle est désormais la réception offerte par les différents acteurs au principe d'accountability par le truchement du comply or explain, devenue la clef de voûte de l'approche retenue par les institutions communautaires en matière de gouvernement d'entreprise.

\section{Historique du comply or explain : la régulation douce britannique}

Le concept «comply or explain » est né outre-Manche, comme réponse aux scandales (Maxwell Communications, Polly Peck et BCCI) qui avaient entaché les milieux d'affaires britanniques. Avec pour objectif initial de préserver les intérêts des actionnaires, la formulation historique du comply or explain s'inscrit dans une vision actionnariale de la firme telle que développée par la théorie positive de l'agence (MacNeil et Li, 2006). L'expression apparaît, pour la première fois, en 1992, dans le rapport Cadbury qui consacre l'entrée de la gouvernance d'entreprise dans le droit international des sociétés cotées (Fasterling et Duhamel, 2009) et émet un certain nombre de recommandations prenant la forme d'un Code de bonnes pratiques. En termes pratiques, le mécanisme du comply or explain oblige les sociétés cotées (ici, sur le London Stock Exchange) à déclarer dans quelle mesure elles appliquent les recommandations des codes de bonne gouvernance et/ou à rendre compte des possibles écarts aux dispositions évoquées par ces mêmes codes.

L'approche britannique, que l'Europe fera sienne, est en matière de régulation des pratiques de gouvernance différente de l'approche légaliste des États-Unis, consacrée par le vote du Sarbanes-Oxley Act (2002). Au lieu d'une réglementation prescriptive, le Royaume-Uni a préféré une forme de régulation douce, pour apporter aux actionnaires la garantie que leurs capitaux soient bien gérés, par l'adoption d'un outil de la transparence, destiné à faire respecter des normes de conduite non contraignantes. L'énoncé du principe d'action de comply or explain contient, dans son essence, une approche volontaire et flexible caractéristique de la conception britannique de la régulation (MacNeil et Li, 2006). Le Combined Code of Corporate Governance, publié en juin 1998, consolide les travaux et recommandations du rapport Cadbury et Greenbury de 1995 : l'objectif historique était d'inciter les entreprises à adopter l'esprit plus que la lettre du code (Arcot et al., 2010). Les travaux précités montrent toutefois que le code a encouragé beaucoup plus la « compliance » (conformité) que l'explication. La logique du mécanisme qui repose sur la faculté de ne pas appliquer ou d'appliquer partiellement un code explique ce manquement. 


\section{La référence à des codes de bonnes pratiques standardisées}

L'obligation de rendre des comptes, selon la méthode du comply or explain, s'établit au regard des recommandations composant les codes de gouvernance. Aguilera et Cuervo-Cazurra (2004) voient dans le rapport de la Business Roundtable, publié en 1978 aux États-Unis, le premier code de gouvernance connu à ce jour (Wirtz, 2006). La véritable diffusion des codes de gouvernance en Amérique du Nord, puis en Europe date des années 1990. Ces codes furent à l'initiative des acteurs du marché boursier, des associations de managers et d'organismes gouvernementaux (Aguilera et Cuervo-Cazurra, 2004).

$\mathrm{Si}$ ces codes peuvent revêtir différentes appellations - codes de meilleures pratiques, de référence, de bonne conduite, de bonne gouvernance, etc. - ils laissent tous entendre, qu'en matière de gouvernance, existent des pratiques modèles et une conduite idéale (Wirtz, 2006). Or, tels qu'ils sont émis et diffusés à l'échelle internationale (cf. Ploix, 2003, p. 131-135), les codes de bonnes pratiques renvoient à un ensemble de critères standardisés dont la définition généralisable à un grand nombre d'entreprises semble difficile (Moris, 2012, p. 148). Conçue à l'origine pour répondre de façon flexible aux caractéristiques du marché britannique et aux particularismes sectoriels, la logique du comply or explain devait introduire dans le champ de la gouvernance l'idée qu'un seul modèle de bonnes pratiques ne pouvait répondre à toutes les situations particulières (Couret, 2010). Néanmoins, cette logique a rapidement accompagné la promotion de bonnes pratiques de gouvernance standardisées (Aguilera et Cuervo-Cazurra, 2004), renforçant au niveau international une tendance à la convergence des modèles (Aguilera et al., 2008). Toutefois, si les critères présents dans les codes prennent une dimension universelle, ils n'en ont dans le fond que les atours, puisqu'ils traduisent une conception particulière de la gouvernance, celle de la gouvernance actionnariale, d'origine anglo-américaine (Aguilera et al., 2008). Malgré les critiques qui peuvent lui être formulées (Segrestin et Hatchuel, 2011), celle-ci demeure un modèle de gouvernance de référence pour les sociétés cotées (Albouy, 2011).

\section{II - LA GOUVERNANCE ACTIONNARIALE RENFORCÉE APPAREMMENT PAR LA DIFFUSION DU COMPLY OR EXPLAIN}

Formulée au début des années 1990, au Royaume-Uni, comme réponse aux pratiques de gouvernance d'entreprise défaillantes, la procédure du comply or explain a traversé la Manche pour se diffuser, à travers la promotion des institutions européennes, à la plupart des systèmes de gouvernance continentaux. Mécanisme clef d'un modèle de gouvernance actionnariale, le concept « comply or explain » est devenu, en Europe, un « principe structurant du gouvernement d'entreprise » (Poulle, 2011, p. 131).

\section{Essaimage en Europe du concept « comply or explain »}

Comme le rappelle Poulle (2011), les réflexions menées outre-Manche en matière de gouvernance ont eu une influence déterminante sur les choix opérés par les institutions communautaires au début des années 2000. Une première orientation à 
l'échelle européenne fut prise à la suite de l'étude comparative et exhaustive du cabinet Weil, Gotshal \& Manges LLP sur 35 codes européens (Weil, Gotshal \& Manges, 2002): l'étude ayant révélé de grandes similarités entre les codes, les institutions européennes ont préféré renoncer au projet d'élaboration d'un code unique de gouvernance européen. La Commission européenne confia alors à un groupe de travail présidé par Jaap Winter la réalisation d'un rapport sur la gouvernance d'entreprise. Publié en 2002, le rapport Winter préconisait le recours au mécanisme du comply or explain ${ }^{3}$. Allant dans le sens de cette recommandation, «les institutions communautaires ont ainsi privilégié la méthode par rapport au contenu en matière de gouvernement d'entreprise » (Poulle, 2011, p. 131). Le régulateur européen a introduit le concept de comply or explain, en 2005, dans le cadre du Forum européen sur le gouvernement d'entreprise en précisant que ce principe devenait la base de l'approche européenne du gouvernement d'entreprise fondé sur des codes (Vignial et MaisonBlanche, 2006). Il faut, toutefois, attendre la publication de la directive 2006/46/CE du Parlement européen et du Conseil du 14 juin 2006, pour que soit rendue « obligatoire une déclaration de gouvernement d'entreprise au regard du code de référence, ou même dans les cas de choix de pratiques allant au-delà des exigences requises par le droit national » et que soit confortée la logique de comply or explain (IFA, 2013, p. 3).
La base de l'approche européenne du gouvernement d'entreprise repose sur la transparence et se traduit par une obligation d'information issue de la directive 2006/46/CE. Entre droit dur (hard law) et droit souple (soft law), selon les cultures juridiques, le compromis préconisé par la méthodologie du comply or explain s'est imposée dans tous les États (Roquilly et Collard, 2009 ; Tchotourian, 2011). L'idée était d'engager la responsabilité des conseils d'administration ou comités d'audit créés ad hoc, devant les actionnaires. La flexibilité de la mesure devait être ainsi contrebalancée par l'effectivité plus grande donnée aux droits des actionnaires, notamment à l'information. L'économie générale du dispositif repose in fine sur l'hypothèse d'un marché de capitaux suffisamment mature pour influencer et réguler le jeu des acteurs économiques (IFA, 2013, p. 11).

Quelques années après la transposition de la directive européenne, le Livre vert de la Commission européenne «Cadre de la gouvernance d'entreprise dans l'UE » (15 avril 2011) considère que l'approche comply or explain, souple et adaptée à toute forme d'entreprise, en fonction de sa taille ou de son secteur d'activité, contribue à « la responsabilisation des entreprises, en les encourageant à vérifier si leurs pratiques de gouvernance sont appropriées et en leur fixant un objectif $»^{4}$. La méthodologie tend à se généraliser à l'ensemble du reporting relatif à la RSE. En lien avec l'OCDE et l'Union européenne, la Global Reporting Initiative (GRI) préconise une formule

3. « La Commission devrait bientôt publier une recommandation aux États membres pour les inciter à intégrer à leur droit des sociétés ou à leurs codes nationaux de gouvernement d'entreprise des règles efficaces dans ce domaine, dont la mise en œuvre relèverait au minimum de l'application du principe "se conformer ou se justifier" $)$ (rapport Winter, 2002, p. 13).

4. Livre vert de la Commission européenne « Cadre de la gouvernance d'entreprise dans l'UE » (2011, p. 21). 
similaire : « report or explain why not » en matière de performance $\mathrm{ESG}^{5}$.

Toutefois, la Commission européenne n'exclut pas des mesures plus contraignantes que de simples recommandations pour réaliser certains ajustements ou protéger davantage les actionnaires minoritaires. $\mathrm{La}$ France a privilégié, quant à elle, le cadre légal de la soft law.

\section{Réception du mécanisme du comply or explain en France}

Si Zattoni et Cuomo (2008) ont montré que les pays de tradition de droit civil, parmi lesquels figure la France, ont adopté plus tardivement les codes de gouvernance que les pays de la «Common Law », la plupart des spécialistes français relativisent ce retard en identifiant l'année 1995 - année de publication du premier rapport Viénot $^{6}$ - comme celle de l'entrée décisive des grandes entreprises françaises dans le débat de la gouvernance (Magnier, 2008 ; Wirtz, 2008). Les premiers acteurs mobilisés en France par la diffusion et l'adoption de pratiques de bonne gouvernance furent, en effet, les dirigeants de grandes entreprises françaises, aux côtés de l'organisation patronale du CNPF et de l'Association française des entreprises privées (Afep). Ce sont ces mêmes acteurs qui ont, jusqu'en 2002 - année de publication du rapport Bouton - promu un socle de «meilleures pratiques » de gouvernance au sein du sys- tème français, et ce, avant que le processus de formalisation de celles-ci ne change et qu'un processus de consolidation soit mis en place avec la publication du rapport Medef $^{7}$ en 2003 (Wirtz, 2008).

Bien que les dirigeants français insistent dès 1995 dans le rapport Viénot 1 sur la formulation d'une gouvernance à la française mobilisée autour de la poursuite d'un objectif d' " intérêt social $»^{8}$, l'analyse des principaux codes de gouvernance français montre que nous sommes ici en présence de « textes promoteurs des standards d'une gouvernance à l'anglo-saxonne » (Wirtz, 2008, p. 69). L'émergence et l'évolution de la référence au principe de comply or explain dans ces mêmes textes révèle un alignement progressif des codes français sur le modèle de gouvernance actionnariale. Notons que l'esprit du principe de comply or explain n'apparaît que dans le rapport Viénot 2 (1999) tandis que sa traduction en langue française (conformer ou expliquer) est pour la première fois mentionnée dans le rapport Afep-Medef 2003. Toutefois, il faut attendre la publication du rapport Afep-Medef de 2008 pour que soit citée en langue anglaise la référence au principe de comply or explain.

C'est l'article 26 de la loi no 2008-649 du 3 juillet 2008 qui intègre pour la première fois le mécanisme du comply or explain dans le dispositif législatif français, en contraignant les entreprises cotées à pro-

\footnotetext{
5. «Input by the Global Reporting Initiative (GRI) to the European Multistakeholder Forum on CSR », Plenary Meeting, 29-30 novembre 2010.

6. Rapport du groupe de travail présidé par Marc Viénot, ancien président directeur générale de la Société générale. 7. Le Conseil national du patronat français s'est transformé et a donné naissance au Mouvement des entreprises de France (Medef) en 1998.

8. «Dans les pays anglo-saxons, l'accent est principalement mis sur l'objectif de maximisation rapide de la valeur de l'action, alors que, sur le continent européen et en particulier en France, il est plutôt mis sur l'intérêt social de l'entreprise. » (rapport Viénot, 1995, p. 8).
} 
duire, chaque année, une "déclaration de conformité au code de gouvernement d'entreprise ». La loi française exige une référence au code de bonne conduite élaboré par les organisations représentatives des entreprises auquel le rapport du président du conseil d'administration (article L. 225-37 alinéa $7 \mathrm{du}$ code de commerce) ou du conseil de surveillance (article L. 225-68 alinéa 8 du même code) devront se référer : «Lorsqu'une société se réfère volontairement à un code de gouvernement d'entreprise élaboré par les organisations représentatives des entreprises, le rapport prévu au présent article précise également les dispositions qui ont été écartées et les raisons pour lesquelles elles l'ont été. Se trouve de surcroît précisé le lieu où ce code peut être consulté. Si une société ne se réfère pas à un tel code de gouvernement d'entreprise, ce rapport indique les règles retenues en complément des exigences requises par la loi et explique les raisons pour lesquelles la société a décidé de n'appliquer aucune disposition de ce code de gouvernement d'entreprise. Le rapport prévu au présent article précise aussi les modalités particulières relatives à la participation des actionnaires à l'assemblée générale ou renvoie aux dispositions des statuts qui prévoient ces modalités. [...] Le rapport prévu au présent article est approuvé par le conseil d'administration (ou le conseil de surveillance) et est rendu public. »

La loi $\mathrm{n}^{\circ} 2010-788$ du 12 juillet 2010 portant engagement national pour l'environnement, dite loi Grenelle II, par un juste parallélisme des formes, demande également aux investisseurs institutionnels (sociétés de gestion, OPCVM, SICAV, etc.) de rendre des comptes sur leurs choix d'investisse- ments selon des critères ESG, par rapport à un code français de transparence ISR (investissement socialement responsable). L'article L. 533-22 du code monétaire et financier renvoie alors aux dispositions règlementaires pour les modalités de l'accountability au cœur de la réforme française sur la sécurité financière par application du système du comply or explain. L'article D. 533-16-1 du code monétaire et financier issu du décret $n^{\circ} 2012-132$ du 30 janvier 2012 énonce ainsi paragraphe deux que certaines sociétés de gestion de portefeuilles doivent donner une «description de la manière dont les résultats de l'analyse sur des critères relatifs au respect d'objectifs sociaux, environnementaux et de qualité de gouvernance sont intégrés dans le processus d'investissement et de désinvestissement; le cas échéant, description de la manière dont les valeurs non appréciées sur la base de ces critères sont prises en compte » et ce, sur le site internet de la société de gestion, et dans le rapport annuel de chaque organisme de placement collectif en valeurs mobilières géré. L'article ajoute in fine « Ces informations peuvent être présentées selon un code élaboré par une association professionnelle. Dans ce cas, la société de gestion précise en préambule le code retenu. "

Mais, ces obligations légales ou règlementaires à l'encontre des émetteurs de titres financiers ou des investisseurs institutionnels ne sont assorties d'aucune sanction juridique : l'absence d'information ou les écarts notifiés ne sont pas susceptibles d'engager la responsabilité de leurs auteurs. De plus, bien que publics, les rapports sur la gouvernance ne sont pas assimilés à une « information financière réglementée » 
selon l'article 2 de la directive "Transparence », (2004/109/CE). Les directives subséquentes n'ont, pour le moment, pas modifié ce point. Tout au plus appartientil au commissaire aux comptes de délivrer « une attestation de l'établissement des autres informations requises aux articles L. 225-37, L. 225-68 et L. 226-10-1, ou, à défaut, le signalement de l'irrégularité constituée par l'absence de certaines de ces informations »; et le cas échéant, de formuler « ses observations sur le caractère manifestement incohérent des autres informations ». Or, sans obligation faite aux entreprises d'informer les publics concernés, le marché ne peut pas jouer son rôle convenu dans le dispositif; pas même une sanction économique ne peut donc être prise, en pratique, sur ce fondement, ce que les études portant sur le contexte britannique confirment (Arcot et al., 2010 ; MacNeil et Li, 2006).

Sur le fond l'AMF note, certes, une amélioration dans les pratiques en raison d'une appréciation exigeante du principe « appliquer ou expliquer » mais c'est en jouant, de facto, le rôle de régulateur des marchés et en vérifiant l'exhaustivité et la qualité de l'information'. L'AMF n'exerce pas un véritable contrôle de régularité des déclarations et elle ne dispose pas non plus d'un pouvoir d'injonction. Sans intervenir sur les contenus, l'AMF se contente d'informer les entreprises émettrices du relevé des mauvaises pratiques, voire demande des rectifications en amont, ce qui conduit certaines sociétés à modifier leurs pratiques, sous la pression. Et elle recommande aux agences de conseil en vote ("proxy advisors ») de publier sur leur site internet leur méthodologie et d'éclairer sur leurs politiques de vote, notamment lorsqu'elles n'exercent pas leurs droits dans l'intérêt exclusif des actionnaires ou porteurs de parts d'organismes collectifs en valeurs mobilières (recommandation 2011-06). Par cette exigence de transparence, l'AMF compte également sur les investisseurs, pour faire respecter les codes de gouvernance, par leurs stratégies de votes, qui elles-mêmes sont fondées sur un code élaboré par la profession. À défaut, c'est le code français de la transparence AFG et FIR de décembre 2009 (déclinaison d'un code ISR européen) qui est recommandé.

Les pratiques demeurent en définitive peu encadrées, même si la démarche n'est plus une simple option, dès lors qu'il existe un code de référence, car l'obligation de se conformer à un code privé de gouvernement d'entreprise est bien prescrite. Néanmoins, ce retrait de l'État au profit d'autorités indépendantes pour assurer exclusivement le contrôle des déclarations n'est pas sans risque.

\section{Expansion du modèle de la gouvernance actionnariale à travers les codes}

En tant que mécanisme pivot des codes de gouvernance, la procédure du comply or explain accentue, de par la contrainte normative qu'elle instaure, les orientations et objectifs de gouvernance contenus dans les recommandations de ces mêmes

9. Cf. Rapport AMF 2012 sur le gouvernement d'entreprise et la rémunération des dirigeants des sociétés cotées et recommandation AMF n 2012-14 (p. 23), ainsi que le rapport AMF 2013 sur l'information publiée par les sociétés cotées en matière de responsabilité sociale, sociétale et environnementale (recommandation $n^{\circ} 1$ de base sur la mise en œuvre du principe « appliquer et s'expliquer »). 
codes (Fasterling et Duhamel, 2009). Qu'il s'agisse de s'y conformer, de les appliquer ou d'expliquer les raisons ayant conduit à s'en écarter, la mise en œuvre du comply or explain confère ainsi une force normative aux critères retenus par les codes. Or, ces derniers dessinent, comme nous l'avons vu précédemment, de façon nette une approche de la gouvernance d'entreprise fondée sur la création de valeur actionnariale (Aguilera et Cuervo-Cazurra, 2004 ; Wirtz, 2006 ; Zattoni et Cuomo, 2008). Ils diffusent ainsi à grande échelle un modèle de gouvernance d'entreprise d'origine anglo-américaine, prévalant autant dans les discours que dans les pratiques (Gomez, 2009; Gehrke et Zarlowski, 2003 ; Moris, 2012).

Berceau du principe de comply or explain, le Royaume-Uni a fait sien, dès l'origine, le modèle anglo-américain de gouvernance actionnariale ; tel est le cas pour le code de référence du Royaume-Uni, le Combined Code of Corporate Governance (MacNeil et Li, 2006). Or, comme le soulignent Arcot et al. (2010), c'est la conception disciplinaire et actionnariale de la gouvernance d'entreprise qui, à travers l'influence du code britannique, a servi de référence aux instances communautaires dans la rédaction de la directive européenne.

La plupart des recommandations issues des codes de gouvernance européens sont non seulement convergentes mais comportent également des éléments s'inspirant fortement de la théorie de l'agence, plus particulièrement des écrits de M.C. Jensen et de ses co-auteurs (Wirtz, 2006 ; Zattoni et Cuomo, 2008 ; Poulle, 2011). On peut voir dans cette diffusion à succès des codes, l'influence d'une «représentation dominante de la valeur actionnariale $»$ : les codes de gouvernance s'alignant sur cette représentation auraient emporté plus de conviction de la part des acteurs concernés (Wirtz, 2006, p. 256).

Parce qu'il induit une présomption «au terme de laquelle les principes contenus dans les codes de gouvernance développent "les bonnes pratiques", "la bonne gouvernance", uniforme et adaptable à toute structure », le mécanisme de comply or explain fait de la gouvernance un « concept d'ordre normatif» (Fasterling et Duhamel, 2009, p. 134). Or, en droit une présomption crée un renversement de la charge de la preuve : une entreprise qui déclare respecter son code ou un code, est donc supposée respecter la loi... Véhiculé à travers les codes, le modèle de la gouvernance actionnariale tend par conséquent, par l'effet normatif du comply or explain, à s'imposer comme modèle de gouvernance dominant (Gomez, 2009). Pour autant, compte tenu des pratiques observées, il n'est pas certain que les relations entre actionnaires et dirigeants soient améliorées.

\section{III - LE GLISSEMENT VERS UN CONCEPT NORMATIF DE GOUVERNANCE OCCULTANT LE PRINCIPE DE RESPONSABILITÉ}

L'application du comply or explain, avec la publication corrélative des motifs sur les écarts éventuels, entretient un mode d'autorégulation, où chaque partie prenante se renvoie en miroir, l'évocation d'une bonne gouvernance ou d'une gouvernance adaptée ou encore « raisonnable » (référentiel Middlenext, cahier $n^{\circ} 2,2009$ ) sur fond de codes imbriqués les uns dans les autres, dont les exigences sont souvent bien adoucies. Ces mesures de soft law peuvent faire perdre de vue la finalité du système, et plus 
grave, entretenir l'illusion, par déplacement de l'objet, que le reporting peut devenir une fin en soi. Par ailleurs, l'application du code n'est jamais qu'un succédané de la loi, pour promouvoir les meilleures pratiques de gouvernance dans un contexte précis. La mise en place de l'outil, par le biais du « comply or explain», peut finir par faire oublier l'objectif premier de responsabilité incombant à l'entreprise. La puissance normative des codes de gouvernance est renforcée, ce qui pose une question de légitimité et des problèmes de cohérence donc d'efficience : par code interposé, les entreprises disposent de la loi comme elles l'entendent. Peut-on encore parler de logique de conformité face à des normes aussi « molles »?

\section{Une nouvelle forme de normativité entre conformisme et conformité}

Le principe d'action du comply or explain entre dans un processus de développement du droit souple ou mou. Cette nouvelle forme de normativité ne conduit pas pour autant à une logique de conformité, quel que soit le pays de réception.

Le déploiement du modèle français est pour le moins original car il démontre le cas échéant la difficulté dans un pays de Civil Law de se départir complètement d'un substrat légal ou réglementaire. Il se traduit par la formule suivante : «Apply or explain, and if you apply, comply or explain » (Fasterling et Duhamel, 2009, p. 136). Et il impose effectivement une hiérarchie très précise : on peut choisir de ne pas s'appuyer sur un code de gouvernement d'entreprise,

Figure 1 - Les obligations françaises en matière de code de gouvernement d'entreprise

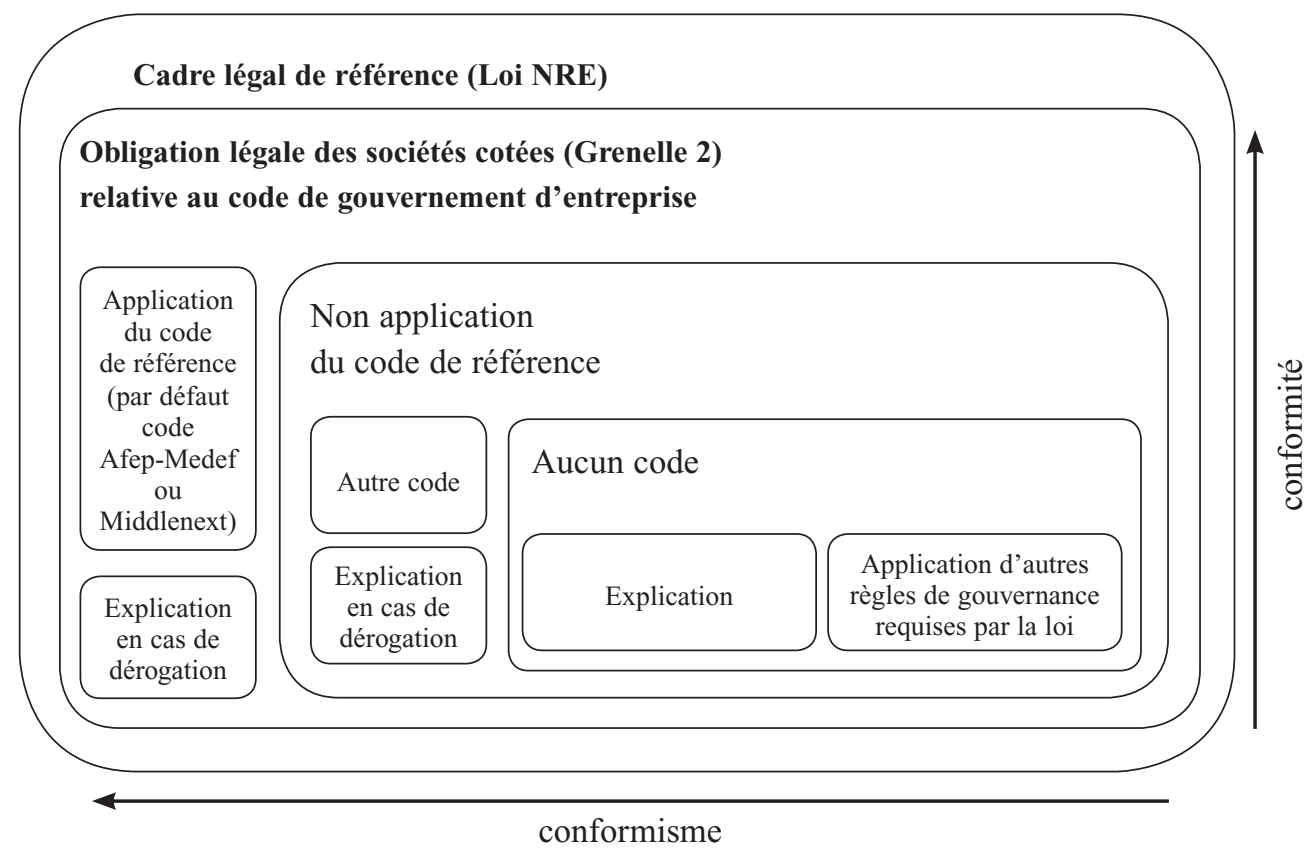


mais il faut alors expliquer les règles adoptées en complément des exigences requises par la loi (première branche); ou alors, si on applique un code de gouvernance, il faut se conformer à ce code ou s'expliquer (deuxième branche). Il ne s'agit pas d'une obligation de conformité pure et simple. La loi prend un caractère supplétif et non subsidiaire, ce que Deumier (2009, p. 165) nomme « la force normative optionnelle ». La contrainte devient relative. Pour autant l'obligation de s'expliquer sur les dérogations, ou les écarts devrait tempérer cette affirmation (Magnier, 2008).

Dans le rapport sur les entreprises faisant référence au code de gouvernance Middlenext pour l'exercice 2011 (rapport IFGE, avril 2013) et le quatrième rapport annuel sur le code Afep-Medef de l'exercice 2011 (décembre 2012) figurent la liste des entreprises ayant adhéré à un code, le nombre d'entreprises répondantes et des exemples précis d'explications fournies en cas de non-application ou d'écarts. On note ainsi, sur le panel recensé, que le taux d'administrateurs indépendants est de $75 \%$ pour les sociétés du CAC 40, $66 \%$ pour les sociétés du SBF : la marge de progression est encore conséquente, sans compter le taux d'abstention et le fait que l'analyse s'établit à partir des déclarations unilatérales de l'entreprise. La parité est mieux respectée dans les conseils d'administration au fil des ans mais la loi l'impose au-delà des codes de gouvernance...

Rien ou presque, le défaut d'explication ou le caractère mensonger des déclarations n'étant que rarement sanctionné, n'assure "l'intériorisation de ces normes et donc leur respect » (Dionne-Proulx et Larochelle, 2010, p. 42). Les codes sont établis en marge du droit. Censés aller au- delà du respect de la légalité stricto sensu, ils n'en sont souvent que la réplique. Pour autant la France fait partie des bons élèves. Néanmoins, bon nombre de rapports et travaux sur la mise en œuvre de l'outil de reporting «comply or explain» par les sociétés cotées en Europe (IFA 2013 ; Livre vert 2011 ; rapport AMF, 2012 ; MacNeil et Li, 2006 ; Arcot et al., 2010) notent des distorsions importantes dans la qualité des explications, quand bien même sont elles fournies. La force régulatrice des conduites et des pratiques fondée sur la persuasion ou la dissuasion crée une «illusion de transparence » (Roquilly et Collard, 2009, p. 7), au mieux une « transparence conformiste » (Duhamel et Fasterling, 2009).

La sanction du marché était donc l'effet affiché et non réellement escompté. Tout a été mis en œuvre, pour éviter les sanctions par différents biais psychologiques (étude pionnière de Fanto, 2002, cité par Wirtz, 2006). Car la loi consacre comme norme une référence à un code qui autorise en fait toutes les dérogations : il n'y a plus alors de différence entre l'obligation de faire et le laissez-faire, le caractère impératif et facultatif, l'obligation de rendre des comptes et l'autorisation de déroger à la règle... La norme juridique devient, par ce tour de prestidigitation, superfétatoire, dès lors que l'entreprise refuse d'engager sa responsabilité au titre d'un code de gouvernement. Ce n'est pas un hasard si Magnier (2008) évoque une réforme en « trompe l'œil ». Et qu'est-ce qu'une norme qui, en toute licéité, met, sur un pied d'égalité ceux qui se conforment et ceux qui contreviennent aux règles? Il n'y a plus de transgression au sens de violation de la règle puisqu'il est permis de ne pas respecter le code ou de s'expliquer, en toute légalité, sur 
la légitimité de ne pas le respecter: le mécanisme du comply or explain est donc une déviance, un conflit d'interprétation, affrontement entre deux conceptualisations rivales de l'action (Ogien, 1995, cité par Babeau et Chanlat, 2008). Généralisé, il sape les fondations de tout système normatif et comporte même une inversion des principes juridiques.

\section{Le risque de confondre le principe d'action (comply or explain) avec le principe de responsabilité (accountability)}

Le glissement sémantique qui s'opère avec l'éclosion d'un concept normatif de gouvernance est symptomatique de cette cristallisation sur les moyens qui conduit à occulter la visée de toutes ces mesures de régulation. En effet, un certain nombre d'auteurs emploient indifféremment le terme «principe » concernant le comply or explain plutôt que démarche, processus ou système. Mais ce raccourci est un abus de langage qui cache une véritable convention. La méthode du comply or explain est au mieux un principe d'action pour les sociétés cotées afin de répondre de leurs actes. C'est l'un des instruments de la transparence, préconisé pour rendre des comptes, fondement de la responsabilité. Le principe général de responsabilité est reconnu dans de nombreuses conventions internationales. Il a valeur, en France, de principe constitutionnel ${ }^{10}$, autrement dit, il est classé dans la hiérarchie des normes traditionnelles, symbolisée par une pyra-

Figure 2 - Risque d'inversion des principes normatifs

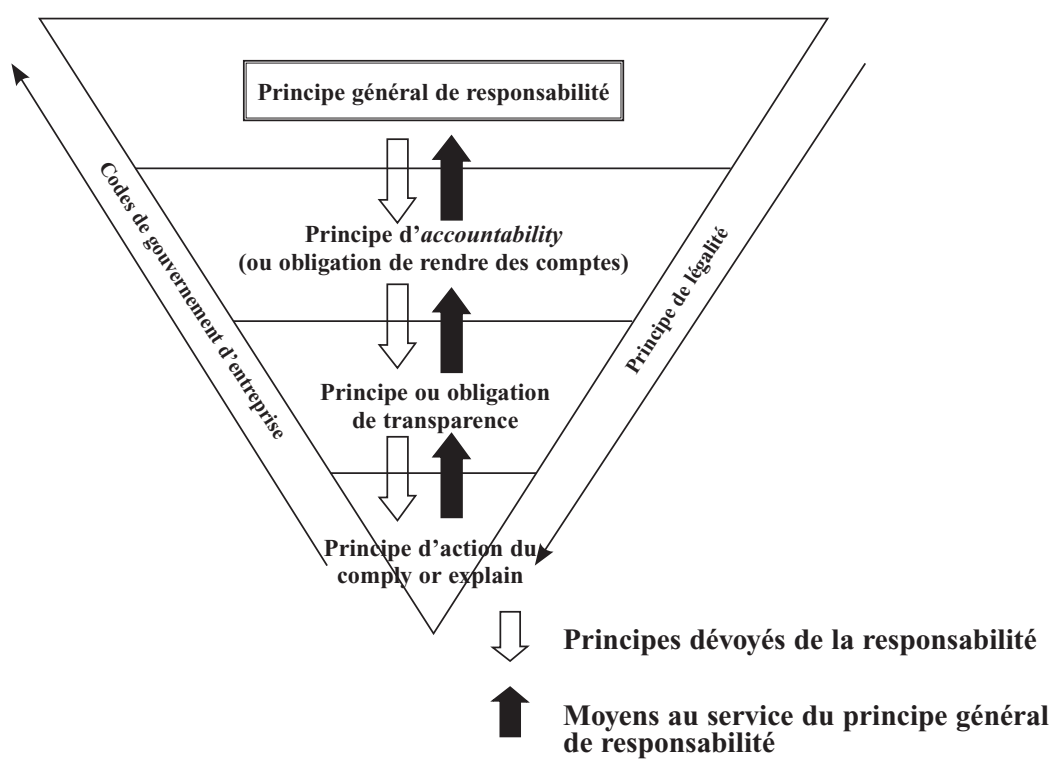

10. Décision du Conseil constitutionnel no ${ }^{\circ} 2-144$ DC du 22 octobre 1982, loi relative au développement des institutions représentatives du personnel. 
mide (la pyramide de Kelsen) au-dessus des lois. Ainsi, les entreprises connaissent des déclinaisons de ce principe de responsabilité à travers des textes de loi ou de règlement, qui sont des normes publiques, impératives, de rang inférieur, directement applicables. Les normes publiques sont dites descendantes. Pour autant, avec le développement de ce que l'on appelle la soft law, ou régulation publique souple, des normes d'origine privée, ont pris le relais et tentent de s'imposer dans le système normatif, pas seulement comme compléments nécessaires, mais également en lieu et place de la norme publique. Ainsi en est-il des codes de gouvernement d'entreprise, référentiels privés créés par des organisations, situés en bas de la pyramide, puisqu'ils sont, en principe, soumis au droit, mais parviennent, grâce à un jeu de pouvoirs des acteurs économiques, à intervertir la hiérarchie des normes. La cohérence apparente des «principes », reliés les uns aux autres, masque de surcroît de nombreux effets pervers : la remise en cause du principe d'universalité de la loi, la mutation des obligations en principes, l'affaissement du pouvoir politique et démocratique, l'institutionnalisation de nouveaux lieux de production de normes, la conformité volontaire substituée à l'obligation d'appliquer, et surtout l'inversion des valeurs. Si les principes sont de rang équivalent, par nature, ce n'est pas le cas des normes, dont la validité est conditionnée au respect de la norme supérieure. C'est ce renversement de la pyramide des valeurs, des principes et des normes que nous avons représenté dans la figure 2.

L'accountability est une forme de responsabilité qui a connu un tel essor que la traduction française, dans la Norme ISO 26000 relative à la responsabilité sociétale des organisations, a donné naissance au néologisme « redevabilité » qui représente un des sept principes de responsabilité sociétale. Mais la redevabilité (« être en mesure de répondre de ses impacts sur la société, l'économie et l'environnement », art. 4.2) n'est qu'une des dimensions de la responsabilité sociétale, comme la transparence, le respect de la loi ou le comportement éthique.

Ces nouveaux principes constituent des modalités, non la finalité de la responsabilité. Or, en surestimant la portée de l'un des instruments de la transparence, tel le modèle de comply or explain, celui-ci agit au contraire, comme un filtre de la transparence; de même, en se concentrant sur l'application des codes, de plus en plus soft, la loi passe par un prisme déformant. Ainsi, par le détournement de l'objet de la surveillance, l'interversion entre la fin et les moyens donc la dégradation des obligations légales, le principe général de responsabilité finit par être totalement occulté. «La référence croissante à des principes à épithète comme fondement de l'expression d'une soft law (principe d'accountability, d'inclusivness, d'indépendance, de prudence, de subsidiarité, de traçabilité, et de transparence) concourent à la mise en exergue d'une impossible responsabilité. » (Pesqueux, 2011, p. 100-101). En effet, le mot « principe » utilisé, à des fins pratiques, sert à propulser au premier rang une norme qui ne devrait pas y figurer, pour empêcher de fournir un critère de distinction entre la règle ordinaire et le principe, ce qui intellectuellement et moralement est critiquable. Ces principes ont souvent un rôle technique. Ils ne peuvent, sauf idéologie, prétendre devenir un principe fondateur 
(Jestaz, 2005, p. 23-25). Pour éviter toute confusion, nous ne rangeons donc pas le comply or explain dans les «principes» mais dans les «principes d'action». En érigeant le comply or explain en principe, la norme prend le pas sur la référence légale. L'instance de régulation n'est plus l'État mais les sociétés cotées, qui décident librement de fixer le curseur de leurs responsabilités. "Se soumettre ou se disculper " (Champaud et Danet, 2008), tel est l'intérêt masqué de nombreux adeptes du comply or explain. Cette inversion traduit «l'aveu d'un État dépassé par son droit » (Magnier, 2008). La régulation autonome trouve ici ses limites par rapport à une régulation de contrôle.

\section{Les limites de la gouvernance par la soft law et les codes de gouvernance}

Le potentiel «régulatoire» de la compliance, comme nouvelle force normative, non (encore) cadrée, touche sa limite. Il résonne chez les juristes, par son apparence de conformité au droit comme l'élément matériel qui prendrait le pas sur l'élément psychologique pour démontrer une responsabilité fondée exclusivement sur les preuves d'application d'un code au détriment parfois de son effectivité ou de son efficacité voire de sa réalité. L'épreuve du droit, nous apprend qu'on ne peut dissocier élément matériel et élément psychologique. Avec cynisme, l'analyse économique du droit pourrait se satisfaire de cet état de fait : l'absence de contrainte juridique, avec un code qui se placerait idéalement audessus de la loi remplirait bien sa mission d'efficacité économique avec une idéologie de la valeur actionnariale au sommet. Mais, dans cette perspective, il y a complet découplage entre le discours affiché et la réalité économique : personne n'est responsable de ses actes. Le principe d'action serait une mystification, l'accountability un mythe.

Or, le principe d'action du comply or explain est fondé sur la liberté avant tout. Vouloir être libre sans exercice de la responsabilité, ou plus exactement, libéré des contraintes juridiques n'est pas une utopie, c'est une déviance. Sans fondement normatif explicite ou déterminable, quelle responsabilité est encourue?

Sous la pression de l'opinion publique, marquée par de nombreux scandales financiers, le gouvernement français avait promis d'intervenir pour plafonner les rémunérations des dirigeants sociaux. Mais suite à une mission d'information sur la transparence de la gouvernance des grandes entreprises, dans le rapport $n^{\circ} 737$ présenté le 20 février 2013 à l'Assemblée nationale par JeanMichel Clément et Philippe Houillon, qui avaient pourtant préconisé l'instauration d'une obligation de se référer à un code et de sanctionner les manquements en cas de violation de ce code (p. 16), c'est finalement l'autorégulation, très critiquée dans sa légitimité et dépourvue d'instruments de contrôle efficients, qui a été confortée (p. 19). En validant le système des codes de gouvernance régulé par des organismes privés, le véritable contrôle de conformité à la loi et par l'État est donc esquivé. Le sous-système normatif qui s'est établi est loin des idéaux affichés, de référentiels qui engageraient les entreprises « à appliquer des standards qui vont au-delà des exigences légales et réglementaires » (rapport AfepMedef, 2012, préface, Pringuet et Parisot, p. 3). Souvent mal appliqués, peu réactualisés, rarement ouverts à d'autres parties prenantes, ces codes exigent aujourd'hui des explications «compréhensibles, perti- 
nentes et circonstanciées 》 (révision code Afep-Medef, 2013, p. 32). Et le principe d'action du comply or explain verrouille ce système d'autocontrôle.

La soft law considère cependant qu'elle crée un principe circulaire d'efficacité. Chatelin-Ertur et Onnée (2009, p. 656) démontrent que l'environnement légal des entreprises se structure autour d'une interaction constante entre droit souple et droit dur, considérant le droit souple à la fois comme un préalable, un complément ou sous influence du droit dur. L'effectivité est liée ou contrainte par l'enchevêtrement qui en résulte, le processus de normalisation en matière de codes de gouvernance s'établissant en définitive par une sélection naturelle entre les meilleures pratiques.

Mais il apparaît que, dans ce cadre, le mécanisme du comply or explain confine à une technique «d'asservissement » de la règle juridique (Bonneau, 2012, p. 135) puisqu'il va jusqu'à marchander la loi, par le biais des codes de gouvernance qui servent de paravent face à un contentieux judiciaire éventuel ou du moins qui créent l'illusion du respect du droit. La contrainte légale est de toute évidence à ce stade insuffisante pour permettre aux actionnaires de disposer de l'information nécessaire pour exercer leurs droits: la sanction économique par le marché n'est donc pas au rendez-vous. Formulée à l'origine pour préserver les intérêts des actionnaires, la logique du comply or explain est détournée dans sa mise en œuvre de son objectif historique et fait courir au modèle actionnarial de la gouvernance un risque de fragilisation. Il faut ici souligner les limites de la portée des modèles et des théories qui ne peuvent donner que ce qu'ils ont, face à des pratiques qui leur échappent et peuvent se retourner contre eux (Albouy, 2012). Ni conformité, ni explication parfois, telle est la réalité. Comment souhaiter, dans ces conditions, l'application généralisée de cette technique de la soft law, au-delà des codes de gouvernance, comme norme de reporting de toutes les informations extrafinancières des entreprises?

\section{CONCLUSION}

Un récent rapport public au gouvernement français présente vingt propositions pour renforcer la démarche de responsabilité sociale des entreprises (RSE) et précise qu'un projet de directive rendant obligatoire la publication d'informations extrafinancières pour les entreprises domiciliées en Europe sera prochainement soumis au Parlement et au Conseil de l'Union européenne (Brovelli et al., 2013). Deux propositions (10 et 18) évoquent, dans le cadre de cette directive, la «logique » du comply or explain notamment comme modalité de l'accountability du dialogue avec les parties prenantes: les entreprises visées auront l'obligation, en cas de non-présentation des informations prescrites, de se justifier. Or, comme nous l'avons démontré, sous couvert de diffusion de bonnes pratiques de gouvernance, le dispositif du comply or explain n'est pas neutre : parce qu'il bouleverse les équilibres normatifs fondamentaux, il dessert plus qu'il ne sert le modèle de la gouvernance actionnariale pour lequel il était conçu à l'origine. L'extension du dispositif de comply or explain au modèle de gouvernance partenariale, ne réduit pas pour autant le risque d'ineffectivité de l'accountability.

Effectivement, en décodant tout le système de fonctionnement du comply or explain 
par rapport aux exigences de l'accountability, cette recherche interdisciplinaire, droit et gestion, a pu mettre en lumière le degré d'irresponsabilité auquel peut aboutir un encastrement de principes instaurés par la pratique. Parce qu'il substitue à la loi une obligation de se soumettre à des normes privées, des codes de gouvernement d'entreprise parfois autoproclamés, sans véritable contrôle, ni du contenu du code, ni de son application pour peu qu'on déclare une conformité de principe, le mécanisme du comply or explain confine au cercle vicieux : à l'absence de sanction s'ajoute un défaut d'objet. C'est l'instrumentalisation des outils de gestion et du droit, au détriment de leur effectivité, qui est alors le plus à redouter pour l'avenir des sociétés anonymes. La puissance principielle du mécanisme en atteste. Comment inverser la tendance ?

Légiférer encore, et toujours plus, n'est pas la solution adéquate, mais c'est à l'État qu'il appartient de trouver les moyens efficaces de sanctionner les comportements qui ne sont pas conformes au droit. De toute évidence, la soft law engendre des zones de non-droit qui répandent l'irresponsabilité de manière épidémique. La logique du comply or explain dénuée de toute sanction juridique efficace apparaît, en effet, comme une mystification. À ce jeu, l'injonction légale ressemble fort à une injonction paradoxale où finalement la raison se perd: on ne sait plus, de la copie (code) ou de l'original (loi), ce que les sociétés doivent appliquer, ni surtout pourquoi, ces règles s'appliquent. Le résultat est davantage une uniformisation des pratiques de transparence (Fasterling et Duhamel, 2009) qu'une application conforme des codes, au point que la méthodologie du comply or explain est érigée en principe, ce qui sème la confusion avec l'objectif d'accountability recherché. Elle peut conduire la gouvernance dans une impasse en favorisant des pratiques, prenant les apparences de la transparence, alors que le principe d'action comply or explain n'est que l'avatar de l'accountability.

\section{Bibliographie}

Aguilera R., Filatotchev I., Gospel H., Jackson G. (2008). "Contingencies, complementarities, and costs in corporate governance models", Organization Science, vol. 19, $\mathrm{n}^{\circ} 3$, p. 475-492.

Aguilera R. et Cuervo-Cazurra A. (2004). "Codes of good governance worldwide: What is the trigger ?", Organization Studies, vol. 25, n³, p. 415-443.

Albouy M. (2012). " La plus belle théorie financière ne peut donner que ce qu'elle a », Revue française de gestion, vol. 38, $\mathrm{n}^{\circ}$ 228-229, p. 107-126.

Albouy M. (2011). "Autorité de gestion et avaries communes : une note de lecture », Finance, Contrôle Stratégie, vol. 14, n 4, p. 7-19.

Arcot S., Bruno V. et Faure-Grimaud A. (2010). "Corporate governance in the UK: Is the comply or explain approach working?", International Review of Law and Economics, vol. $30, \mathrm{n}^{\circ} 2$, p. $193-201$. 
Babeau O. et Chanlat J.-F. (2008). «La transgression, une dimension oubliée de l'organisation », Revue française de gestion, vol. 34, n 183, p. 201-219.

Bonneau Th. (2012). «Le juriste et le droit des marchés financiers : la technique juridique asservie ? », La place du juriste face à la norme, tome XVI, Thèmes \& Commentaires, Association H. Capitant, Dalloz, p. 135-149.

Bréchet J.-P., Monin Ph. et Saives A.-L. (2008). « Légitimité, déviance et délit : Des défis pour le management stratégique ", Revue française de gestion, vol. 34, n 183, p. 15-34.

Brovelli L., Drago X. et Molinié E. (2013). 20 propositions pour renforcer la démarche de responsabilité sociale des entreprises (RSE), Rapport public au Gouvernement.

Cadbury Committee (1992). The report of the committee on the financial aspects of corporate governance.

Champaud C. et Danet D. (2008). «Chronique sociétés en général », Revue trimestrielle de droit commercial, $\mathrm{n}^{\circ} 1$ à 4 .

Charreaux G. et Desbrières P. (1998). « Gouvernance des entreprises : valeur partenariale contre valeur actionnariale », Finance Contrôle Stratégie, vol. 1, n 2, p. 57-88.

Chatelin-Ertur C. et Onnée S. (2009). « Des forces normatives des Codes de gouvernance de entreprises à la puissance normative du paradigme en économie organisationnelle », La force normative, Naissance d'un concept, C. Thibierge et al. (dir.), LGDG-Bruyland.

Couret A. (2010). "The development of the 'comply or explain' principle in Europe", Annual meeting of the SASE Annual conference, Temple University, Philadelphia, PA, USA, 24 juin.

Deumier P. (2009). « La force normative optionnelle », La force normative, naissance d'un concept, ouvrage collectif, Thibierge C. et al. (dir.), LGDJ, Éd. Bruylant, p. 165-170.

Dionne-Proulx J. et Larochelle G. (2010). "Éthique et gouvernance d'entreprise », Management \& Avenir, $\mathrm{n}^{\circ} 32$, p. 36-53.

Dumez H. (2013). Méthodologie de la recherche qualitative, Vuibert.

Dumez H. (2008). « De 1'obligation de rendre des comptes ou accountability », Gérer et Comprendre, $\mathrm{n}^{\circ}$ 91, mars, p. 4-8.

Fasterling B. et Duhamel J.-C. (2009). « Le comply or explain : la transparence conformiste en droit des sociétés », Revue internationale de droit économique, vol. 23, $\mathrm{n}^{\circ} 2$, p. 129-157.

Gehrke I. et Zarlowski P. (2003). «La diffusion de la valeur actionnariale en France : une lecture néo-institutionnaliste », Comptabilité Contrôle Audit, numéro spécial, mai, p. 189-205.

Gomez P.Y. (2009). « La gouvernance actionnariale et financière, une méprise théorique », Revue française de gestion, vol. 35, $\mathrm{n}^{\circ}$ 198-199, p. 369-391.

Hedström P. et Bearman P. (2009). The Oxford handbook of analytical sociology, Oxford, Oxford University press.

Institut français des administrateurs (IFA), "Comply or explain ». Guide pratique de mise en ouvre, 2013.

Jestaz Ph. (2005). Les sources du droit, coll. « Connaissance du droit », Dalloz. 
Livre vert de la Commission européenne (2011). « Cadre de la gouvernance d'entreprise dans l'UE », 15 avril.

MacNeil I. et Li X. (2006). “'Comply or explain': Market discipline and non-compliance with the combined code", Corporate Governance: An International Review, vol. 14, $\mathrm{n}^{\circ} 5$, p. 486-496.

Magnier V. (2008). « Le principe "se conformer ou s'expliquer", une consécration en trompe l'œil ? », La Semaine Juridique - Entreprise et Affaires, LexisNexis, n 23, 5 juin, p. 3-5.

Moris K. (2012). «Les enjeux de la gouvernance d'entreprise aujourd'hui. De meilleures théories pour de meilleures pratiques », Revue française de gestion, vol. 38, n 228-229, novembre-décembre, p. 147-166.

Mulgan R. (2000). “Accountability': an Ever-Expanding Concept?”, Public Administration, vol. 78, n 3, p. 555-573.

Pesqueux Y. (2012). "La comptabilité peut-elle être éthique?», RIMHE - Revue interdisciplinaire management homme(s) et entreprise, $n^{\circ} 1$, mars/avril, p. 97-110.

Pesqueux Y. (2011). « Pour une approche essentialiste des valeurs dans l'organisation », Le management par les valeurs, Bergery L. (dir.), Éd. Hermès Science, coll. « Management, Société et Technologie », Lavoisier, p. 89-120.

PesqueuxY.(2010). « Organisation innovatrice et responsabilité sociale et environnementale », $8^{\circ}$ Congrès de l'ADERSE, La Rochelle.

Ploix H. (2003). Le dirigeant et le gouvernement d'entreprise, Pearson Education.

Poulle J.-B. (2011). Le principe « se conformer ou expliquer » en droit boursier, thèse de doctorat en Droit, sous la dir. de Pietrancosta A.

Rapport AMF sur le gouvernement d'entreprise et la rémunération des dirigeants des sociétés cotées, 2012.

Rapport Gomez et Guedri sur les entreprises faisant référence au code de gouvernance Middlenext pour l'exercice 2011, IFGE, avril 2013.

Rapport Winter «Un cadre réglementaire moderne pour le droit européen des sociétés », 4 novembre 2002, Rapport du Groupe de haut niveau d'experts en droit des sociétés.

Roquilly Ch. et Collard Ch. (2009). « De la conformité réglementaire à la performance : pour une approche multidimensionnelle du risque juridique », Centre de recherche LegalEdhec, septembre.

Segrestin B. et Hatchuel A. (2011). " Autorité de gestion et avaries communes : pour un complément de droit de l'entreprise », Finance Contrôle Stratégie, vol. 14, n 2, juin, p. 9-36.

Tchotourian Y. (2011). «Le droit au secours du risque de mauvaise gouvernance : regard d'un juriste français sur les réformes récentes du Code belge des sociétés en matière de corporate governance », Management \& Avenir, $\mathrm{n}^{\circ}$ 41, janvier, p. 140-155.

Vignial A. et Maidon-Blanche C. (2006). "Comply or Explain”, Lettre de l'administrateur, IFA, $\mathrm{n}^{\circ} 11$, octobre, p. 8 .

Weil, Gotshal \& Manges LLP (2002). Comparative study of corporate governance codes relevant to the European Union and its Member States, Commission européenne. 
Wirtz P. (2008). « L'attrait psychologique des codes de "meilleures pratiques" en France », Revue française de gestion, vol. 34, $\mathrm{n}^{\circ} 183$, avril, p. 51-71.

Wirtz P. (2006). " "Meilleures pratiques" de gouvernance et création de valeur: une appréciation critique des codes de bonne conduite », chapitre 9, Gouvernance des Entreprises. Nouvelles perspectives, Charreaux G. et Wirtz P., Economica.

Zattoni A. et Cuomo F. (2008). "Why adopt codes of good governance? A comparison of institutional and efficiency perspectives", Corporate Governance: An International Review, vol. 16, n 1, p. 1-15. 
